Tropical Journal of Pharmaceutical Research March 2021; 20 (3): 483-489

ISSN: $1596-5996$ (print); 1596-9827 (electronic)

(C) Pharmacotherapy Group, Faculty of Pharmacy, University of Benin, Benin City, 300001 Nigeria.

Available online at http://www.tjpr.org

Original Research Article

http://dx.doi.org/10.4314/tjpr.v20i3.6

\title{
MiR-483-3p exacerbates pediatric pneumonia by suppressing IGF1 expression in alveolar macrophage
}

\author{
Jie Feng ${ }^{1}$, Jingjing Tang ${ }^{1}$, Pingping Liu ${ }^{2}$, Xiaolan Zhang ${ }^{2 *}$ \\ ${ }^{1}$ Department of Pediatrics, The People's Hospital of Fenghua Ningbo, Ningbo City, Zhejiang Province 315500, ${ }^{2}$ Department of \\ Pediatrics, Jiangning Hospital Affiliated to Nanjing Medical University, Nanjing City, Jiangsu Province 211100, China \\ *For correspondence: Email: Ippsss66@163.com, xiaolanzhang188@126.com; Tel: +86-025-52281848, +86-025-52281848
}

\begin{abstract}
Purpose: To investigate the action of miR-483-3p in pediatric pneumonia and identify potential biomarkers.

Methods: Bronchoalveolar lavage was collected from 38 pneumonia patients and 25 healthy children. The expression of miR-483-3p in the lavage was determined using qRT-PCR. Alveolar macrophages collected from lavage of healthy children were cultured and used for functional assays. Transwell assay was conducted to evaluate macrophage cell migration. Cell viability and apoptosis were evaluated in lipopolysaccharide (LPS)-induced human pulmonary alveolar epithelial cells (HPAEpiCs) by CCK8 (cell counting kit - 8) or flow cytometry, respectively.

Results: MiR-483-3p was significantly elevated in bronchoalveolar lavage of pneumonia patients, when compared to healthy children $(p<0.001)$. MiR-483-3p, which targets insulin-like growth factor 1 (IGF1), decreased the mRNA and protein expression of IGF1 in alveolar macrophages collected from the lavage of healthy children. MiR-483-3p reduced motility of macrophages. IGF1 counteracted the LPSinduced decrease in cell viability and the increase in apoptosis of HPAEpiCs. Conditioned medium from macrophages transfected with miR-483-3p inhibitor increased cell viability and reduced cellular apoptosis of LPS-induced HPAEpiCs. However, conditioned medium from macrophages transfected with miR-483-3p mimics decreased cell viability and increased apoptosis.

Conclusion: MiR-483-3p negatively regulates IGF1 to promote progression of pediatric pneumonia, providing a potential therapeutic target in pediatric pneumonia.
\end{abstract}

Keywords: MiR-483-3p, Insulin-like growth factor (IGF1), TNBC, Alveolar macrophage, Pediatric pneumonia

\begin{abstract}
This is an Open Access article that uses a fund-ing model which does not charge readers or their institutions for access and distributed under the terms of the Creative Commons Attribution License (http://creativecommons.org/licenses/by/4.0) and the Budapest Open Access Initiative (http://www.budapestopenaccessinitiative.org/read), which permit unrestricted use, distribution, and reproduction in any medium, provided the original work is properly credited.

Tropical Journal of Pharmaceutical Research is indexed by Science Citation Index (SciSearch), Scopus, International Pharmaceutical Abstract, Chemical Abstracts, Embase, Index Copernicus, EBSCO, African Index Medicus, JournalSeek, Journal Citation Reports/Science Edition, Directory of Open Access Journals (DOAJ), African Journal Online, Bioline International, Open-J-Gate and Pharmacy Abstracts
\end{abstract}

\section{INTRODUCTION}

Pneumonia, a common respiratory disease caused by pathogens or viral infection, is the leading cause of mortality and morbidity in children [1]. Although pneumonia is preventable via surgical and medical treatment, pediatric pneumonia often leads to poor prognosis and numerous severe complications, due to its high rate of recurrence [1]. Therefore, an effective therapeutic strategy for pediatric pneumonia would facilitate treatment. 
Alveolar macrophages, located at the interface between lung tissue and air, are the first line of defense against microorganisms [2]. Inhibition of apoptosis of alveolar macrophages contributes to the survival of mice and rats with Pneumocystis pneumonia [3]. Therefore, alveolar macrophages play an important role in the development of pediatric pneumonia. Research has shown that macrophages secrete cytokines to participate in alveolar epithelial cell injury during pneumonia [4]. Insulin-like growth factor 1, secreted by macrophages, regulates immunometabolic challenge [4], and promotes neurogenesis and nerve sensitization during endometriosisassociated pain [5]. Recently, macrophagederived IGF1 has been shown to mediate phagocytosis in response to inflammation [6]. Suppression of IGF1 made newborn mice more susceptible to pneumonia [7]. Thus, alveolar macrophage-derived IGF1 may be an effective target for the treatment of pediatric pneumonia.

A recent study suggested the dysregulation of miRNA expression in pneumonia [8], and the miRNA-mRNA network may be a potential therapeutic strategy for pediatric pneumonia [8]. For example, miR-155 was involved in the immune response during pneumonia [9]. Previous studies have shown that influenza virus infection leads to upregulation of miR-483-3p in pulmonary exosomes, and promotes the inflammatory response [10]. Because miR-483 has been shown to be upregulated in peripheral blood of patients with pneumonia [11], the present study was conducted to investigate the potential role and mechanism of miR-483-3p in pediatric pneumonia, thus shedding light on a potential therapeutic benefit for pediatric pneumonia.

\section{EXPERIMENTAL}

\section{Study population and bronchoalveolar lavage}

Thirty-eight pneumonia patients and 25 healthy children were recruited at the People's Hospital of Fenghua Ningbo. Written parental informed consent was acquired from all the volunteers. The current study was approved by the Ethics Committee of the People's Hospital of Fenghua Ningbo (approval no. 2018112), and conducted in accordance with the 1964 Helsinki Declaration and its later amendments for ethical research involving human subjects [12]. For the collection of bronchoalveolar lavage, $100 \mathrm{~mL}$ sterile isotonic saline, in $20 \mathrm{~mL}$ aliquots, was instilled into the right middle lobe via fiberoptic bronchoscope. Bronchoalveolar lavage fluid was aspirated with gentle suction, and then filtered through sterile gauze. After centrifugation at 500 $\times g$ for $15 \mathrm{~min}$, the cells were harvested for further analysis.

\section{Collection and transfection of alveolar macrophages}

Bronchoalveolar lavage cells of healthy children were suspended in RPMI-1640 medium (Lonza, Basel, Switzerland) containing $10 \%$ fetal bovine serum (Gibco, Waltham, MA, USA). The cells (5 $\times 10^{5} /$ well) were seeded into plates and cultured at $37{ }^{\circ} \mathrm{C}$ with $5 \% \mathrm{CO}_{2}$ for $1 \mathrm{~h}$. After removing non-adherent cells, the adherent alveolar macrophages were identified via esterase staining. The isolated alveolar macrophages were transfected with miR-418-3p mimics, inhibitor, or the corresponding negative controls (NC mimic and NC inhibitor), via Lipofectamine 3000 (Invitrogen, Carlsbad, CA, USA).

\section{Determination of IGF1}

Insulin-like growth factor 1 levels in alveolar macrophage culture medium were determined via IGF1 antibody DuoSet (R\&D Systems, Minneapolis, MN, USA), following the manufacturer' $s$ instructions.

\section{Luciferase reporter assay}

The sequence of IGF1 3'-UTR, or the mutant sequence, was subcloned into psiCHECK2 (Promega, Madison, WI, USA). Alveolar macrophages were co-transfected with miR-483$3 p$ mimics or NC mimic (GenePharma, Suzhou, China) and psiCHECK2-wt-IGF1 or psiCHECK2mut-IGF1. Forty-eight hours later, luciferase activities were measured with the Dual Luciferase Assay Kit (Promega).

\section{Transwell assay}

Alveolar macrophages suspended in RPMI 1640 medium were placed in the upper compartment of vitronectin-coated chambers (BD Biosciences, Bedford, MA, USA). RPMI 1640 medium with no addition, or with $100 \mathrm{ng} / \mathrm{mL}$ M-CSF, $100 \mathrm{ng} / \mathrm{mL}$ MCP-1, or $100 \mathrm{ng} / \mathrm{mL}$ fMLP, was added to the lower chamber. Three hours later, numbers of macrophages per high-power field that had migrated into the lower compartment were determined.

\section{Quantitative reverse transcription PCR (qRT- PCR)}

Total RNA from bronchoalveolar lavage cells or macrophages was extracted via Trizol, and reverse-transcribed into cDNA. TB Green Premix Ex Taq (Takara, Dalian, China) was used to 
perform the qRT-PCR analysis. GAPDH and U6 were used as endogenous controls. The primer sequences are shown in Table 1.

\section{Cell culture and treatment}

HPAEpiC, purchased from ScienCell Research Laboratories (San Diego, CA, USA), were cultured in RPMI 1640 medium containing $10 \%$ fetal bovine serum at $37{ }^{\circ} \mathrm{C}$ with $5 \% \mathrm{CO}_{2}$. Cells were incubated for $24 \mathrm{~h}$ with RPMI-1640 medium with or without LPS (10 $\mathrm{mg} / \mathrm{L})$ or recombinant human IGF1 (1 ng/mL), prior to other assays.

Samples of conditioned medium from macrophages transfected with miR-483-3p mimics or inhibitor were collected via centrifugation at $2500 \mathrm{rpm}$ for $30 \mathrm{~min}$. The LPSinduced HPAEpiC cells were incubated with a $1: 1$ mixture of the conditioned medium and RPMI 1640. Cell viability and apoptosis were determined as below.

\section{Cell counting}

After different treatments, HPAEpiC were seeded into plates for $24 \mathrm{~h}$, and then incubated with CCK8 solution (Cell Counting Kit-8, Dojindo, Tokyo, Japan) at $37{ }^{\circ} \mathrm{C}$ for $1 \mathrm{~h}$. Absorbance at $450 \mathrm{~nm}$ was measured with a Microplate Autoreader (Thermo Fisher, Waltham, MA, USA).

\section{Cellular apoptosis}

HPAEpiC cells suspended in $150 \mu \mathrm{L}$ binding buffer were incubated with $5 \mu \mathrm{L}$ PI $(100 \mu \mathrm{g} / \mathrm{mL})$ and $1 \mathrm{U} / \mathrm{ml}$ ribonuclease (KeyGEN BioTech, Jiangning, Nanjing, China). Cells were then incubated with $5 \mu \mathrm{L}$ fluorescein isothiocyanateconjugated annexin V. Cells were analyzed by FACS flow cytometry (Attune, Life Technologies, Darmstadt, Germany).

\section{Statistical analysis}

Data are expressed as mean \pm standard deviation. Statistical analyses were performed using one-way analysis of variance and
Student's t-test, as appropriate. Values of $p<$ 0.05 were considered statistically significant.

\section{RESULTS}

Up-regulation of miR-483-3p in
$\begin{aligned} & \text { bronchoalveolar lavage of pediatric } \\ & \text { pneumonia patients }\end{aligned}$

To investigate the relationship between miR-483$3 p$ and pneumonia, expression of $\mathrm{miR}-483-3 p$ in bronchoalveolar lavage was evaluated by qRTPCR. Results showed higher expression of miR$483-3 p$ in bronchoalveolar lavage of pediatric pneumonia patients than in healthy children (Figure 1), suggesting that miR-483-3p might regulate pediatric pneumonia.

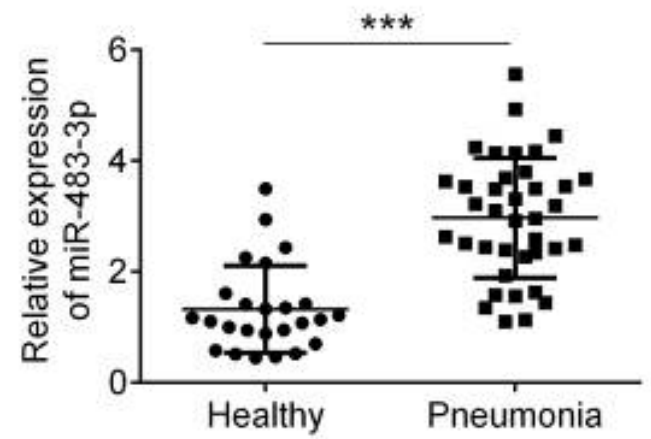

Figure 1: Up-regulation of miR-483-3p in bronchoalveolar lavage of pediatric pneumonia patients. Expression of miR-483-3p in bronchoalveolar lavage fluid of pneumonia patients and healthy children was evaluated by qRT-PCR; ${ }^{* * *} p<0.001$

\section{Validation of miR-483-3p target binding}

To further investigate the role of miR-483-3p in pediatric pneumonia, the target gene of miR-483$3 p$ was determined in isolated alveolar macrophages. Insulin-like growth factor $1 \mathrm{mRNA}$ contains a potential binding site for miR-483-3p (Figure 2A). To validate the ability of miR-483-3p to bind IGF1, a luciferase reporter assay was conducted.

Table 1: Primer sequences for PCR

\begin{tabular}{ll}
\hline ID & Sequence (5'- 3') \\
\hline U6 F & CTCGCTTCGGCAGCACA \\
U6 R & AACGCTTCACGAATTTGCGT \\
miR-418-3p F & ACACTCCAGCTGGGTCCAACATTGTCTTTAG \\
miR-418-3p R & TGGTGTCGTGGAGTCG \\
IGF1 F & GCTCTTCAGTTCGTGTGTGG \\
IGF1 R & CGCAATACATGTCCAGCCTC \\
GAPDH F & ACCACAGTCCATGCCATCAC \\
GAPDH R & TCCACCACCCTGTTGCTGTA \\
\hline
\end{tabular}


The result showed that miR-483-3p mimics decreased luciferase activity of cells expressing psiCHECK2-wt-IGF1 (Figure 2 B), while not significantly affecting the expression of psiCHECK2-mut-IGF1 (Figure $2 \mathrm{~B}$ ), in which the site of miR-483-3p binding in IGF1 was mutated (Figure $2 \mathrm{~A}$ ).

Alveolar macrophages were transfected with either miR-483-3p mimics or inhibitor to assess the regulation of IGF1 expression by miR-483-3p (Figure $2 \mathrm{C}$ ). The mRNA expression of IGF1 was decreased in macrophages transfected with miR483-3p mimics (Figure 2 D) and increased by miR-483-3p inhibitor (Figure 2D). Moreover, considering that macrophages secrete IGF1, the levels of IGF1 protein were then evaluated in culture medium from macrophages transfected with miR-483-3p mimics or inhibitor. Results showed that levels of IGF1 secretion by macrophages were also enhanced by miR-483$3 p$ inhibitor and reduced by miR-483-3p mimics (Figure $2 \mathrm{E}$ ), confirming that miR-483-3p could target and inhibit expression of IGF1 in macrophages.

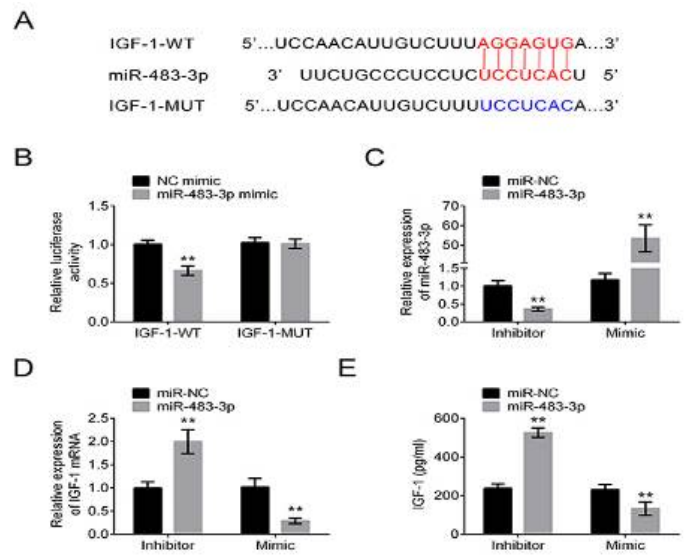

Figure 2: Validation of miR-483-3p target binding. (A) Potential binding site of miR-418-3p in 3'UTR of IGF1. (B) Effect of miR-483-3p mimic on luciferase activity of psiCHECK2-wt-IGF1 or psiCHECK2-mut-IGF1. (C) Transfection efficiency of miR-483-3p mimics or inhibitor in alveolar macrophages. (D) Effect of miR483-3p on mRNA expression of IGF1 in alveolar macrophages. (E) Effect of miR-483-3p on protein expression of IGF1 in alveolar macrophage culture medium; ${ }^{* *} p<0.01$

\section{MiR-483-3p regulated motility of macrophages}

A transwell assay utilizing vitronectin-coated filters was conducted to assess the ability of miR$483-3 p$ to regulate macrophage motility. The results demonstrated that over-expression of miR-483-3p reduced the motility of macrophages in response to the chemotactic factors M-CSF, MCP-1, and fMLP (Figure 3), while knockdown of miR-483-3p promoted macrophage motility (Figure 3).

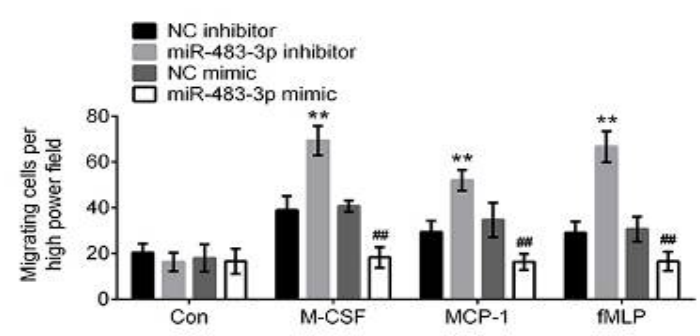

Figure 3: MiR-483-3p regulated motility of macrophages. Effects of miR-483-3p on migration of alveolar macrophages in response to chemotactic factors M-CSF, MCP-1, or FMLP, evaluated by transwell assay; ${ }^{*} \#$ \# $<0.01$

\section{MiR-483-3p promoted apoptosis of LPS- induced HPAEpiC}

A cell-based model of LPS-induced lung injury was established to investigate the role of miR483-3p in pediatric pneumonia. Treatment of HPAEpiC cells with $10 \mathrm{mg} / \mathrm{L}$ LPS decreased cell viability (Figure $4 \mathrm{~A}$ ) and promoted apoptosis (Figure 4 B). Conditioned medium from macrophages transfected with miR-483-3p inhibitor increased cell viability (Figure $4 \mathrm{~A}$ ) and suppressed apoptosis (Figure 4 B) of LPStreated HPAEpiC cells. However, cell viability of LPS-treated HPAEpiC was reduced by conditioned medium from macrophages transfected with miR-483-3p mimics (Figure $4 \mathrm{~A}$ ), and apoptosis was promoted (Figure $4 \mathrm{~B}$ ). In addition, recombinant human IGF1 treatment counteracted the LPS-dependent loss of cell viability in HPAEpiC (Figure $4 \mathrm{~A}$ ), and attenuated LPS-induced apoptosis (Figure $4 \mathrm{~B}$ ), suggesting that miR-483-3p promoted apoptosis of LPSinduced HPAEpiC through regulation of IGF1.

\section{DISCUSSION}

MiRNAs are regarded as critical regulators in lung diseases, including pneumonia [8]. They also function as candidate diagnostic biomarkers of pneumonia [8]. MiR-483 may be implicated in pulmonary hypertension [13], and demonstrates a protective effect against chronic obstructive pulmonary disease [14]. Moreover, miR-483-3p was highly expressed in lungs during viral infection [15], and increased in peripheral blood plasma of pneumonia patients [11]. The present study was therefore conducted to investigate the role and mechanism of miR-483-3p in pediatric pneumonia. 
A

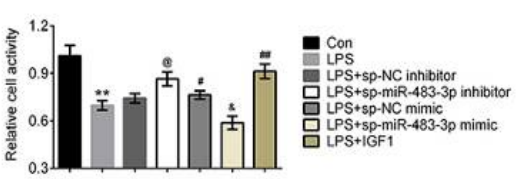

B

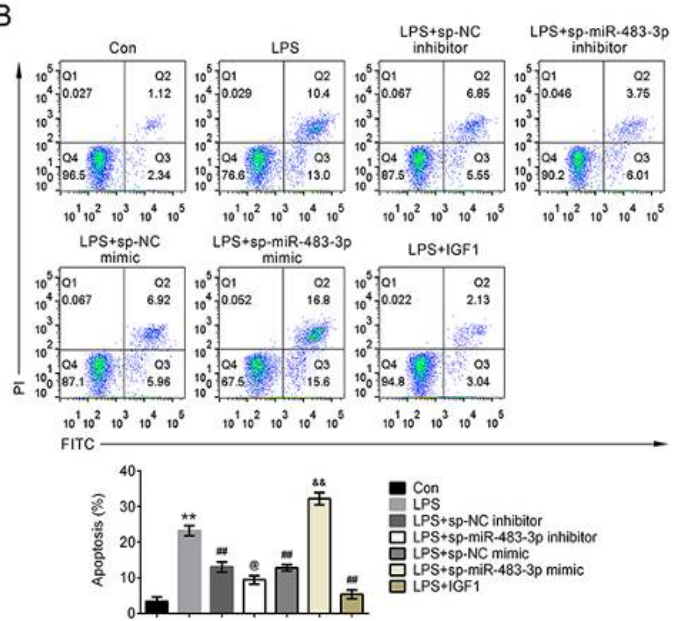

Figure 4: MiR-483-3p promoted apoptosis of LPStreated HPAEpiC cells. (A) Effect of miR-483-3p and IGF1 on cell viability of LPS-induced HPAEpiC. (B) Effect of miR-483-3p and IGF1 on apoptosis of LPSinduced HPAEpiC; @\#\&p $<0.05$. ** \#\#\&\&p $<0.01$

Bronchoalveolar lavage fluid evaluation has been widely regarded as a powerful method for the identification of pathogens of pneumonia [16]. MiRNAs, when delivered into alveolar macrophages, regulate inflammasome activation and macrophage polarization in response to pneumonia [17]. In line with a previous study showing that miR-483-3p was highly expressed in bronchoalveolar lavage fluid exosomes during viral infection [18], the present study demonstrated that miR-483-3p was enhanced in bronchoalveolar lavage of pediatric pneumonia patients.

Alveolar macrophages have chemotactic ability, and play a role in pulmonary host defense, including against pneumonia [3]. Inflammationinduced alteration of alveolar macrophages contributes to lung immunoparalysis, and remodeling of alveolar macrophages contributes to recovery from pneumonia [19]. The present study indicated that miR-483-3p suppressed macrophage motility, contributing to pediatric pneumonia, while miR-483-3p inhibition attenuated pediatric pneumonia through promotion of macrophage motility. Moreover, LPS induces breakdown and hyperpermeability of the pulmonary epithelial barrier [20]; the same study used LPS-induced HPAEpiC as an in vitro cell model of acute lung injury. In our cell model of pneumonia, LPS also induced cell injury of
HPAEpiC, causing loss of cell viability and increased apoptosis. In addition, conditioned medium from macrophages transfected with miR483-3p mimics aggravated LPS-induced cell injury of HPAEpiC, confirming that miR-483-3p might contribute to pediatric pneumonia. In light of the phagocytic [21] or apoptotic [22] capacities of alveolar macrophages to mitigate pneumonia, the roles of miR-483-3p in phagocytosis and apoptosis of alveolar macrophages should be investigated further. In addition, alveolar macrophages are the predominant proinflammatory cells during lung injury initiation, but also participate in resolving the inflammation [23]. Modification of inflammatory responses of alveolar macrophages could help to ameliorate pneumonia [23]. The role of miR-483-3p in macrophage inflammatory responses during pneumonia should also be investigated further.

MiRNA controls pneumonia through binding to the target gene. IGF1 was validated as a target of miR-483-3p, and miR-483-3p inhibits expression of IGF1 in alveolar macrophages. Previous studies have shown that IGF1 is associated with miR-483-3p-mediated polycystic ovarian syndrome [24]. Functional assays showed that IGF1 treatment counteracted the effect of LPS on cell viability of HPAEpiC, and attenuated LPS-induced cell apoptosis, suggesting IGF1 has ameliorative effects in pneumonia. Moreover, conditioned medium from macrophages transfected with miR-483-3p inhibitor attenuated cell injury, with increased cell viability and decreased apoptosis, revealing that miR-483-3p might contribute to pediatric pneumonia through down-regulation of IGF1 in alveolar macrophages.

Low expression of IGF1 contributes to dysfunction of alveolar macrophages [25], and high expression of alveolar macrophage-derived IGF1 results in enhanced phagocytosis of alveolar epithelial cells to control inflammation in asthmatic mice [26]. Since IGF1 decreases inflammatory responses and redirects phagocytic activity of macrophages [6], and IGF1 in alveolar macrophages exerts an antiapoptotic effect in interstitial lung diseases [27], miR-483-3p might contribute to pediatric pneumonia by regulation of alveolar macrophage-derived IGF1-mediated inflammation, apoptosis, and phagocytosis.

\section{CONCLUSION}

MiR-438-3p decreases macrophage motility but promotes LPS-induced cell injury by targeting IGF1, thus providing a potential therapeutic target in pediatric pneumonia. 


\section{DECLARATIONS}

\section{Conflict of interest}

No conflict of interest is associated with this work.

\section{Contribution of authors}

We declare that this work was done by the authors named in this article and all liabilities pertaining to claims relating to the content of this article will be borne by the authors. Jie Feng and Jingjing Tang designed the study, supervised the data collection, and analyzed the data; Pingping Liu interpreted the data and prepare the manuscript for publication; Xiaolan Zhang supervised the data collection, analyzed the data, and reviewed the draft of the manuscript. All authors have read and approved the manuscript.

\section{Availability of data and materials}

All data generated or analyzed during this study are included in this published article.

\section{Open Access}

This is an Open Access article that uses a funding model which does not charge readers or their institutions for access and distributed under the terms of the Creative Commons Attribution License (http://creativecommons.org/licenses/by/ 4.0) and the Budapest Open Access Initiative (http://www.budapestopenaccessinitiative.org/rea d), which permit unrestricted use, distribution, and reproduction in any medium, provided the original work is properly credited.

\section{REFERENCES}

1. Shuangquan $L$, Jianzhong $W$, Hongfeng $L$, Gang $L$, Wenli $S$, Caiyun G, Yuanhui L, Chunqing $W$, Xuelian $Z$, Zhonghou $H$. Combination of thymosin $\alpha 1$ with conventional therapy improves APC and IL-1R1 levels in children with severe pneumonia. Trop J Pharm Res 2018; 17(10): 2093-2099.

2. Chalk K, Meisel C, Spies C, Volk T, Thuenemann K, Linneweber J, Wernecke K-D, Sander M. Dysfunction of alveolar macrophages after cardiac surgery and postoperative pneumonia? an observational study. Critical Care 2013; 17(6): R285.

3. Lasbury ME, Durant PJ, Ray CA, Tschang D, Schwendener R, Lee C-H. Suppression of alveolar macrophage apoptosis prolongs survival of rats and mice with Pneumocystis pneumonia. J Immunol 2006; 176(11): 6443-6453.
4. Spadaro O, Camell CD, Bosurgi L, Nguyen $K Y$, Youm Y$H$, Rothlin CV, Dixit VD. IGF1 shapes macrophage activation in response to immunometabolic challenge. Cell reports 2017; 19(2): 225-234.

5. Forster $R$, Sarginson A, Velichkova A, Hogg C, Dorning A, Horne AW, Saunders PTK, Greaves E. Macrophagederived insulin-like growth factor-1 is a key neurotrophic and nerve-sensitizing factor in pain associated with endometriosis. FASEB J 2019; 33(10): 11210-11222.

6. Han CZ, Juncadella IJ, Kinchen JM, Buckley MW, Klibanov AL, Dryden K, Onengut-Gumuscu $S$, Erdbrügger U, Turner SD, Shim YM et al. Macrophages redirect phagocytosis by non-professional phagocytes and influence inflammation. Nature 2016; 539(7630): 570-574.

7. Oherle K, Acker E, Bonfield M, Wang T, Gray J, Lang I, Bridges J, Lewkowich I, Xu Y, Ahlfeld $S$ et al. Insulin-like Growth Factor 1 Supports a Pulmonary Niche that Promotes Type 3 Innate Lymphoid Cell Development in Newborn Lungs. Immunity 2020; 52(2): 275-294.

8. Zhang $X$, Huang $F$, Yang $D$, Peng $T$, Lu G. Identification of miRNA-mRNA Crosstalk in Respiratory Syncytial Virus-(RSV-) Associated Pediatric Pneumonia through Integrated miRNAome and Transcriptome Analysis. Mediat Inflamm 2020; 2020(1): 8919534.

9. Podsiad A, Standiford TJ, Ballinger MN, Eakin R, Park P, Kunkel SL, Moore BB, Bhan U. MicroRNA-155 regulates host immune response to postviral bacterial pneumonia via IL-23/IL-17 pathway. Am J Physiol-Lung C 2015; 310(5): L465-L475.

10. Xu N, He D, Shao Y, Qu Y, Ye K, Memet O, Zhang L, Shen J. Lung-derived exosomes in phosgene-induced acute lung injury regulate the functions of mesenchymal stem cells partially via miR-28-5p. Biomed Pharmacother 2020; 121: 109603.

11. Huang S, Feng $C$, Zhai Y-Z, Zhou X, Li B, Wang L-L, Chen $W, L v F-Q, L i T-S$. Identification of miRNA biomarkers of pneumonia using RNA-sequencing and bioinformatics analysis. Exp Ther Med 2017; 13(4): 1235-1244.

12. PP R. Human experimentation. Code of ethics of the world medical association. Declaration of Helsinki. Brit Med J 1964; 2(5402): 177-177.

13. Zhang J, He Y, Yan X, Chen S, He M, Lei Y, Zhang J, Gongol B, Gu M, Miao $Y$ et al. MicroRNA-483 amelioration of experimental pulmonary hypertension. EMBO Mol Med 2020; 12(5): e11303.

14. Shen Z, Tang W, Guo J, Sun S. miR-483-5p plays a protective role in chronic obstructive pulmonary disease. Int J Mol Med 2017; 40(1): 193-200.

15. Nguyen TH, Liu X, Su ZZ, Hsu AC-Y, Foster PS, Yang M. Potential Role of MicroRNAs in the Regulation of Antiviral Responses to Influenza Infection. Front Immunol 2018; 9: 1541.

16. Vernikos $P$, Kampolis CF, Konstantopoulos $K$, Armaganidis A, Karakitsos $P$. The Role of Bronchoscopic Findings and Bronchoalveolar Lavage Fluid Cytology in Early Diagnosis of Ventilator-

Trop J Pharm Res, March 2021; 20(3): 488 
Associated Pneumonia. Respir Care 2016; 61(5): 658667.

17. Lee $H$, Groot M, Pinilla-Vera M, Fredenburgh LE, Jin Y. Identification of miRNA-rich vesicles in bronchoalveolar lavage fluid: Insights into the function and heterogeneity of extracellular vesicles. J Control Release 2019; 294: 43-52.

18. Maemura T, Fukuyama S, Sugita Y, Lopes TJS, Nakao $T$, Noda T, Kawaoka Y. Lung-Derived Exosomal miR483-3p Regulates the Innate Immune Response to Influenza Virus Infection. The J Infect Dis 2018; 217(9): 1372-1382.

19. Guillon A, Arafa El, Barker KA, Belkina AC, Martin I, Shenoy AT, Wooten AK, Lyon De Ana C, Dai A, Labadorf $A$ et al. Pneumonia recovery reprograms the alveolar macrophage pool. JCI Insight 2020; 5(4).

20. Xiang Y, Zhang S, Lu J, Zhang W, Cai M, Qiu D, Cai D. USP9X promotes LPS-induced pulmonary epithelial barrier breakdown and hyperpermeability by activating an NF-kappaBp65 feedback loop. Am J Physiol Cell Physiol 2019; 317(3): C534-C543.

21. Bordon Y. Blocking SIRPa could restore macrophage appetite and mitigate pneumonia. Nat Rev Immunol 2020; 20(6): 349-349.

22. Preston JA, Bewley MA, Marriott HM, McGarry Houghton A, Mohasin M, Jubrail J, Morris L, Stephenson YL, Cross S, Greaves DR et al. Alveolar Macrophage Apoptosis-associated Bacterial Killing Helps Prevent
Murine Pneumonia. Am J Respir Crit Care Med 2019; 200(1): 84-97.

23. Long ME, Gong K-Q, Eddy WE, Volk JS, Morrell ED, Mikacenic C, West TE, Skerrett SJ, Charron J, Liles WC. MEK1 regulates pulmonary macrophage inflammatory responses and resolution of acute lung injury. JCl insight 2019; 4(23): e132377.

24. Xiang $Y$, Song $Y$, Li Y, Zhao D, Ma L, Tan L. miR-483 is Down-Regulated in Polycystic Ovarian Syndrome and Inhibits KGN Cell Proliferation via Targeting Insulin-Like Growth Factor 1 (IGF1). Med Sci Monitor 2016; 22:3383-3393.

25. Bessich JL, Nymon AB, Moulton LA, Dorman D, Ashare A. Low levels of insulin-like growth factor-1 contribute to alveolar macrophage dysfunction in cystic fibrosis. J Immunol 2013; 191(1): 378-385.

26. Zhang Y, Chen J, Xue Q, Wang J, Zhao L, Han K, Zhang $D$, Hou L. Prognostic Significance of MicroRNAs in Glioma: A Systematic Review and Meta-Analysis. BioMed Research International 2019; 2019: 40159694015969.

27. Kopiński P, Sladek K, Szczeklik J, Soja J, Szlubowski A, Balicka-Slusarczyk B, Lackowska B, Plato $M$, Szpechcinski A. Expression of insulin-like growth factor-I (IGF-I) in alveolar macrophages and lymphocytes obtained by bronchoalveolar lavage (BAL) in interstitial lung diseases (ILD). Assessment of IGF-I as a potential local mitogen and antiapoptic cytokine. Folia Histochem Cyto 2006; 44: 249-258. 